\title{
Half-Space Neutron Transport with Linearly Anisotropic Scattering
}

\author{
N. J. MCCoRMICK* AND I. KUŠČER* \\ Department of Nuclear Engineering, The University of Michigan, Ann Arbor, Michigan
}

(Received 12 April 1965)

\begin{abstract}
The method developed by Case is used to solve four time-independent, one-speed problems for neutron transport in a homogeneous medium where the scattering function is linear in the cosine of the scattering angle. The solutions to the albedo, Milne, Green's function, and constant isotropic source problems for a half-space are facilitated by the use of half-range bi-orthogonality relations between the eigenfunctions of the homogeneous transport equation. Expressions gre also derived for the emerging angular densities and the densities and net currents on the surface of the half-space.
\end{abstract}

\section{INTRODUCTION}

$\mathrm{T}$ HE Case approach to solving neutron transport problems utilizes an expansion of the neutron angular density in terms of the eigenfunctions of the homogeneous transport equation. The set of eigenfunctions was first shown to be complete for the case of isotropic scattering of one-speed neutrons.' Using these results, answers to many problems were obtained. ${ }^{1-5}$ The completeness theorem for one-speed neutrons was extended to the case of anisotropic scattering by Mika ${ }^{6}$ and explicit results for the Milne problem with linearly anisotropic scattering were found by Shure and Natelson.?

Recently, orthogonality relations between the eigenfunctions were observed ${ }^{8}$ which simplified the solution of one-speed problems with isotropic scattering. An extension to the case of linearly anisotropic scattering was also indicated at that time. It is this approach which is followed here.

An attempt towards further generalization has recently been made. It appears that the eigenfunctions generally obey a set of bi-orthogonality relations of the same form as mentioned in Ref. 8 . If the scattering function is of order $N$ in the cosine of the scattering angle, the eigenfunction $\varphi_{v}(\mu)$ and its "adjoint" $\tilde{\varphi}_{\nu}(\mu)$ differ by a term $\frac{1}{2} c \nu B(\nu, \mu)$,

* Present address: Institute of Physics, University of Ljubljana, Ljubljana, Yugoslavia.

1 K. M. Case, Ann. Phys. (N. Y.) 9, 1 (1960).

2 K. M. Case, Recent Developments in Neutron Transport Theory, Michigan Memorial Phoenix Project Report, The University of Michigan (1961).

M. R. Mendelson and G. C. Summerfield, J. Math. Phys. 5, 668 (1964).

4 G. J. Mitsis, Nucl. Sci. Eng. 17, 55 (1963).

S. J. McCormick and M. R. Mendelson, Nucl. Sci. Eng. 20, 462 (1964).

J. R. Mika, Nucl. Sci. Eng. 11, 415 (1961). (1964)

${ }_{7}$ F. Shure and M. Natelson, Ann. Phys. (N. Y.) 26, 274

${ }_{8}^{8}$ I. Kušcer, N. J. McCormick, and G. C. Summerfield, Ann. Phys. 30, 411 (1964).

N. J. McCormick, "One-Speed Neutron Transport Problems in Plane Geometry," Ph.D. thesis, The University of Michigan (1964). where $B$ is a polynomial of order $(N-1)$ in both variables. A rigorous proof of these two statements is still lacking. In any event, the computation of $B(\nu, \mu)$ would in general be very tedious. Only in two cases is the situation comparatively simple: in that of linearly anisotropic scattering $(N=1)$ and in that of a nonabsorbing medium with $N=2 .^{\circ} \mathrm{We}$ will restrict ourselves to the first case.

Sections II-IV deal with an absorbing medium. After presenting the bi-orthogonality relations (Sec. II), we apply them to four standard half-space problems (Sec. III): the albedo, Milne, Green's function, and constant isotropic source problems. A special calculation (Sec. IV) leads to simplified formulas for the emerging angular densities and related quantities. In Sec. V, the first three problems are solved for a nonabsorbing medium.

\section{DEFINITIONS AND BI-ORTHOGONALITY RELATIONS}

The transport equation to be solved, written in the usual notation, ${ }^{6,7}$ is

$$
\begin{aligned}
& {\left[\mu \frac{\partial}{\partial x}+1\right] \psi(x, \mu)} \\
& \quad=\frac{c}{2} \int_{-1}^{1}\left[1+b \mu \mu^{\prime}\right] \psi\left(x, \mu^{\prime}\right) d \mu^{\prime}+q,
\end{aligned}
$$

where $\psi(x, \mu)$ is the azimuthal integral of the angular density. Here $-1 \leq b \leq 1$, and we choose $c<1$, deferring the case $c=1$ until Sec. V. For the homogeneous equation, separation of variables is achieved through the ansatz

$$
\psi(x, \mu)=e^{-x / \nu} \varphi_{\nu}(\mu),
$$

where the eigenfunctions $\varphi_{\nu}(\mu)$ are normalized such that

$$
\int_{-1}^{1} \varphi_{\nu}(\mu) d \mu=1 .
$$


There are two kinds of eigenfunctions. The continuum modes, belonging to the interval $-1<\nu<1$, are given $b y^{7}$

$$
\begin{gathered}
\varphi_{\nu}(\mu)=\frac{1}{2} c \nu d(\nu \mu) \mathrm{P}\left[(\nu-\mu)^{-1}\right]+\lambda(\nu) \delta(\nu-\mu), \\
\lambda(\nu)=1-\frac{c \nu}{2} \mathrm{P} \int_{-1}^{1} \frac{d(\nu \mu)}{\nu-\mu} d \mu \\
=d\left(\nu^{2}\right)\left[1-c \nu \tanh ^{-1} \nu\right]-b(1-c)^{2} \nu^{2} \\
d(\nu \mu)=1+b(1-c) \nu \mu .
\end{gathered}
$$

[The symbol P in Eq. (4) is a reminder that we must take the Cauchy principal value of any integral over $\nu$ or $\mu$.] Furthermore, there are two discrete modes,

$$
\varphi_{ \pm}(\mu)=\frac{1}{2} c \nu_{0} d\left( \pm \nu_{0} \mu\right) /\left(\nu_{0} \mp \mu\right),
$$

belonging to the two real roots, ${ }^{6} \pm \nu_{0}$, of the equation

$$
\Lambda\left( \pm \nu_{0}\right)=0 \text {, }
$$

where

$$
\begin{aligned}
\Lambda(z) & =1-\frac{c z}{2} \int_{-1}^{1} \frac{d(z \mu)}{z-\mu} d \mu \\
& =d\left(z^{2}\right)\left[1-c z \tanh ^{-1}(1 / z)\right]-b(1-c)^{2} z^{2}
\end{aligned}
$$

It will be useful to know the derivative of $\Lambda(z)$ at $z=\nu_{0}$ :

$$
\Lambda^{\prime}\left(\nu_{0}\right)=\frac{c d\left(\nu_{0}^{2}\right)}{\nu_{0}\left(\nu_{0}^{2}-1\right)}-\frac{(1-c) d\left(3 \nu_{0}^{2}\right)}{\nu_{0} d\left(\nu_{0}^{2}\right)}
$$

We also infer from Eq. (9) that

$$
\Lambda(\infty)=(1-c)\left(1-\frac{1}{3} c b\right) .
$$

The value of $\lambda(\nu)$ is related to the boundary values of $\Lambda(z)$ on the cut $(-1,1)$ by the equation

$$
\begin{aligned}
\Lambda^{ \pm}(\nu) & \equiv \lim _{\epsilon \rightarrow 0^{+}} \Lambda(\nu \pm i \epsilon) \\
& =\lambda(\nu) \pm i \frac{1}{2} \pi c \nu d\left(\nu^{2}\right), \quad-1<\nu<1 .
\end{aligned}
$$

As was pointed out in Ref. 8, instead of orthogonality relations we now have bi-orthogonality relations for the eigenfunctions $\varphi_{p}(\mu)$ and $\varphi_{ \pm}(\mu)$, with "adjoints" of the form

$$
\begin{aligned}
& \tilde{\varphi}_{\nu}(\mu)=\varphi_{\nu}(\mu)+\frac{1}{2} c \nu B, \\
& \tilde{\varphi}_{ \pm}(\mu)=\varphi_{ \pm}(\mu) \pm \frac{1}{2} c \nu_{0} B .
\end{aligned}
$$

These relations are proved in much the same way as those for isotropic scattering, and at the same time the value of the constant $B$ is derived.

The method involves the use of the functions ${ }^{7}$

$$
\begin{aligned}
X(z) & =\frac{1}{1-z} \exp \left[\frac{1}{2 \pi i} \int_{0}^{1} \ln \frac{\Lambda^{+}(\mu)}{\Lambda^{-}(\mu)} \frac{d \mu}{\mu-z}\right], \\
\gamma(\mu) & =\frac{c \mu}{2} \frac{X^{+}(\mu)}{\Lambda^{+}(\mu)}=\frac{c \mu}{2} \frac{X^{-}(\mu)}{\Lambda^{-}(\mu)} \\
& =\frac{1}{2} c \mu\left[\Lambda(\infty)\left(\nu_{0}^{2}-\mu^{2}\right) X(-\mu)\right]^{-1}, \quad 0 \leq \mu \leq 1,
\end{aligned}
$$

and of the moments of the latter,

$$
\gamma_{n}=\int_{0}^{1} \gamma(\mu) \mu^{n} d \mu
$$

In addition, we need the identities ${ }^{7}$

$$
\begin{gathered}
X(z) X(-z)=\Lambda(z) /\left(\nu_{0}^{2}-z^{2}\right) \Lambda(\infty), \\
X\left(\nu_{0}\right) X\left(-\nu_{0}\right)=-\Lambda^{\prime}\left(\nu_{0}\right) / 2 \nu_{0} \Lambda(\infty), \\
X^{2}(0)=1 / \nu_{0}^{2} \Lambda(\infty), \\
\int_{0}^{1} \frac{\gamma(\mu) d\left(\mu^{2}\right)}{\mu-z} d \mu=X(z),
\end{gathered}
$$

$\mathrm{P} \int_{0}^{1} \frac{\gamma(\mu) d\left(\mu^{2}\right)}{\nu-\mu} d \mu$

$$
=-(2 / c \nu) \lambda(\nu) \gamma(\nu), \quad 0<\nu<1,
$$

$$
\begin{gathered}
\int_{0}^{1} \gamma(\mu) d\left(\mu^{2}\right) d \mu=\lim _{s \rightarrow \infty}[-z X(z)]=1 \\
\gamma_{-1}+b(1-c) \gamma_{1}=X(0) \\
d\left(\nu_{0}^{2}\right) d\left(\bar{\nu}^{2}\right)=(1-c) / \gamma_{0}^{2} \Lambda(\infty)
\end{gathered}
$$

where

$$
\bar{\nu}=\gamma_{1} / \gamma_{0} .
$$

These identities help us to show that bi-orthogonality in $0<\mu<1$ among the set $\varphi_{+}(\mu), \varphi_{\nu}(\mu)$, $0<\nu<1$, and the adjoints is produced by the weight function $\left(\nu_{0}-\mu\right) \gamma(\mu)$. We also find that $B$ must be chosen as

$$
B=b(1-c)\left(\nu_{0}-\bar{\nu}\right) / d\left(\nu_{0} \bar{\nu}\right) .
$$

Let us note that the quoted weight function is closely related to Chandrasekhar's $H$-function ${ }^{7}$ :

$$
\left(\nu_{0}-\mu\right) \gamma(\mu)=\frac{1}{2} c \mu[\Lambda(\infty)]^{-1 / 2} H(\mu)
$$

The bi-orthogonality relations, and a set of related formulas useful in applications, are listed below (where $0<\nu<1$ and $0<\nu^{\prime}<1$ ). In order to save space, we use in some of the formulas the symbol $\xi$ for either $\nu^{\prime}$ or $\nu_{0}$. Correspondingly, $\varphi_{\xi}(\mu)$ denotes either a continuum eigenfunction or $\varphi_{+}(\mu)$. Products of two singular eigenfunctions will be understood in the same sense as in Ref. 8.

$$
\begin{aligned}
& \int_{0}^{1} \varphi_{\nu}(\mu) \tilde{\varphi}_{\nu}(\mu)\left(\nu_{0}-\mu\right) \gamma(\mu) d \mu \\
& =\left(\nu_{0}-\nu\right) \gamma(\nu) \Lambda^{+}(\nu) \Lambda^{-}(\nu) \delta\left(\nu-\nu^{\prime}\right) \\
& \int_{0}^{1} \varphi_{\nu}(\mu) \tilde{\varphi}_{+}(\mu)\left(\nu_{0}-\mu\right) \gamma(\mu) d \mu=0
\end{aligned}
$$




$$
\begin{aligned}
& \int_{0}^{1} \varphi_{+}(\mu) \tilde{\varphi}_{,}(\mu)\left(\nu_{0}-\mu\right) \gamma(\mu) d \mu=0, \\
& \int_{0}^{1} \varphi_{+}(\mu) \ddot{\varphi}_{+}(\mu)\left(\nu_{0}-\mu\right) \gamma(\mu) d \mu \\
& =-\left(\frac{1}{2} c \nu_{0}\right)^{2} X\left(\nu_{0}\right) d\left(\nu_{0}^{2}\right), \\
& \int_{0}^{1} \varphi_{-x}(\mu) \tilde{\varphi}_{\xi}(\mu)\left(\nu_{0}-\mu\right) \gamma(\mu) d \mu \\
& =\left(\frac{1}{2} c \nu\right)^{2} \frac{\tilde{\varphi}_{\xi}(-\nu)}{\Lambda(\infty)\left(\nu_{0}-\nu\right) \gamma(\nu)}, \\
& \int_{0}^{1} \varphi_{-}(\mu) \tilde{\varphi}_{\xi}(\mu)\left(\nu_{0}-\mu\right) \gamma(\mu) d \mu \\
& =c \nu_{0} \xi X\left(-\nu_{0}\right) \tilde{\varphi}_{+}(-\xi), \\
& \int_{0}^{l} \varphi_{\xi}(\mu) \tilde{\varphi}_{-x}(\mu)\left(\nu_{0}-\mu\right) \gamma(\mu) d \mu \\
& =\left(\frac{c \nu}{2}\right)^{2} \frac{\varphi_{\xi}(-\nu)}{\Lambda(\infty)\left(\nu_{0}-\nu\right) \gamma(\nu)}, \\
& \int_{0}^{1} \varphi_{-r}(\mu) \tilde{\varphi}_{->}(\mu)\left(\nu_{0}-\mu\right) \gamma(\mu) d \mu \\
& =\frac{c \nu}{2} \frac{1}{\Lambda(\infty)}\left[\frac{c \nu}{2} \frac{\varphi_{\nu^{\prime}}(\nu)}{\left(\nu_{0}-\nu\right) \gamma(\nu)}-\frac{c \nu^{\prime}}{2} \frac{\bar{\varphi}_{\nu^{\prime}}(\nu)}{\left(\nu_{0}-\nu^{\prime}\right) \gamma\left(\nu^{\prime}\right)}\right], \\
& \int_{0}^{1} \varphi_{-}(\mu) \tilde{\varphi}_{-\nu}(\mu)\left(\nu_{0}-\mu\right) \gamma(\mu) d \mu \\
& =\frac{c \nu}{2}\left[\frac{c \nu}{2} \frac{\varphi_{+}(\nu)}{\Lambda(\infty)\left(\nu_{0}-\nu\right) \gamma(\nu)}-2 \nu_{0} X\left(-\nu_{0}\right) \tilde{\varphi}_{+}(\nu)\right], \\
& \int_{0}^{1} \bar{\varphi}_{\xi}(\mu)\left(\nu_{0}-\mu\right) \gamma(\mu) d \mu \\
& =\frac{1}{2} c \xi(1-c) / \Lambda(\infty) \gamma_{0} d\left(\nu_{0} \bar{\nu}\right), \\
& \int_{0}^{1} \tilde{\varphi}_{-r}(\mu)\left(\nu_{0}-\mu\right) \gamma(\mu) d \mu \\
& =\frac{c \nu}{2} \frac{1}{\Lambda(\infty)}\left[\frac{c \nu}{2} \frac{1}{\left(\nu_{0}-\nu\right) \gamma(\nu)}-\frac{1-c}{\gamma_{0} d\left(\nu_{0} \bar{\nu}\right)}\right], \\
& \int_{0}^{1} \tilde{\varphi}_{\xi}(\mu)\left(\nu_{0}-\mu\right) \gamma(\mu) \frac{d \mu}{\mu} \\
& =\frac{1}{2} c[\Lambda(\infty)]^{-1 / 2}(1+B \xi), \\
& \int_{0}^{1} \tilde{\varphi}_{-,}(\mu)\left(\nu_{0}-\mu\right) \gamma(\mu) \frac{d \mu}{\mu} \\
& =\frac{c}{2}\left\{\frac{1-B \nu}{[\Lambda(\infty)]^{1 / 2}}-\frac{c \nu}{2} \frac{1}{\Lambda(\infty)\left(\nu_{0}-\nu\right) \gamma(\nu)}\right\} .
\end{aligned}
$$

Through the use of these relations, we can express the results for typical half-space problems in terms of the functions $X$ or $\gamma$ and the moments of the latter. The numerical evaluation of these functions, for any given $c$ and $b$, may be performed by iteration of the nonlinear integral equation of Shure and Natelson."

\section{SOLUTIONS TO STANDARD HALF-SPACE PROBLEMS}

We wish to determine the angular density for the (a) albedo, (b) Milne, (c) Green's function, and (d) constant isotropic source problems, all for the halfspace $x \geq 0$ and $c<1$. These problems are defined by the following source and boundary conditions:

$$
\begin{gathered}
q= \begin{cases}0, & \text { (a), (b) } \\
\delta\left(x-x_{0}\right) \delta\left(\mu-\mu_{0}\right), & \text { (c) } \\
1, & \text { (d) }\end{cases} \\
{[\psi(x, \mu)]_{x \rightarrow \infty} \rightarrow \begin{cases}\text { bounded, } & \text { (a), (c), (d) } \\
\varphi-(\mu) e^{x / v_{0}}, & \text { (b) }\end{cases} } \\
\psi(0, \mu)=\left\{\begin{array}{lll}
\delta\left(\mu-\mu_{0}\right), & \mu>0, & \text { (a) } \\
0, & \mu>0, & \text { (b) }-(\mathrm{d})
\end{array}\right.
\end{gathered}
$$

The desired solution is expanded in terms of the eigensolutions (4) and (7). If $q \neq 0$, one further term has to be added, namely the solution of the corresponding infinite-medium problem. Thus we see that the conditions (41) and (42) are met by the expansion

$$
\begin{aligned}
\psi(x, \mu)=f(x, \mu)+ & a_{+} \varphi_{+}(\mu) e^{-x / r_{0}} \\
& +\int_{0}^{1} A(\nu) \varphi_{\nu}(\mu) e^{-x / \nu} d \nu
\end{aligned}
$$

where

$$
f(x, \mu)= \begin{cases}0, & \text { (a) } \\ \varphi_{-}(\mu) e^{x / r_{0}}, & \text { (b) } \\ G_{\infty}\left(x_{0}, \mu_{0} \rightarrow x, \mu\right), & \text { (c) } \\ 1 /(1-c) . & \text { (d) }\end{cases}
$$

The function $G_{\infty}\left(x_{0}, \mu_{0} \rightarrow x, \mu\right)$ is the solution to the infinite-medium Green's function problem for linearly anisotropic scattering ${ }^{6}$ :

$$
\begin{aligned}
& G_{\infty}\left(x_{0}, \mu_{0} \rightarrow x, \mu\right) \\
& =2 \varphi_{ \pm}\left(\mu_{0}\right) \varphi_{ \pm}(\mu) e^{-\left|x-x_{0}\right| / \nu_{0}} / c \nu_{0}^{2} \Lambda^{\prime}\left(\nu_{0}\right) d\left(\nu_{0}^{2}\right) \\
& \quad+\int_{0}^{1} \frac{\varphi_{ \pm}\left(\mu_{0}\right) \varphi_{ \pm}(\mu) e^{-\left|x-x_{0}\right| / x}}{\nu \Lambda^{+}(\nu) \Lambda^{-}(\nu)} d \nu, \quad x \gtrless x_{0} .
\end{aligned}
$$

The boundary conditions (43), applied to (44), lead to an equation of the form (for $\mu>0$ )

$$
a_{+} \varphi_{+}(\mu)+\int_{0}^{1} A(\nu) \varphi_{\nu}(\mu) d \nu=\psi(\mu),
$$

from which the expansion coefficients $a_{+}$and $A(v)$ must be determined. Here $\psi(\mu)$ is a known function, namely 


$$
\psi(\mu) \equiv \psi(0, \mu)-f(0, \mu) \psi(\mu)= \begin{cases}\delta\left(\mu-\mu_{0}\right), & \text { (a) } \\ -\varphi_{-}(\mu), & \text { (b) } \\ -G_{\infty}\left(x_{0}, \mu_{0} \rightarrow 0, \mu\right), & \text { (c) } \\ -1 /(1-c) . & \text { (d) }\end{cases}
$$

Use of the half-range bi-orthogonality relations of Sec. II immediately gives the following results:

$$
\begin{aligned}
& a_{+}=R /\left[-\left(\frac{1}{2} c \nu_{0}\right)^{2} X\left(\nu_{0}\right) d\left(\nu_{0}^{2}\right)\right], \\
& {\left[\begin{array}{l}
\left(\nu_{0}-\mu_{0}\right) \gamma\left(\mu_{0}\right) \tilde{\varphi}_{+}\left(\mu_{0}\right), \\
-c \nu_{0}^{2} X\left(-\nu_{0}\right) \tilde{\varphi}_{+}\left(-\nu_{0}\right),
\end{array}\right.} \\
& R=\left\{\begin{array}{l}
-c \nu_{0}^{2} X\left(-\nu_{0}\right) \tilde{\varphi}_{+}\left(-\nu_{0}\right), \\
-\left[X\left(-\nu_{0}\right) \tilde{\varphi}_{+}\left(-\nu_{0}\right) \frac{2 \varphi_{-}\left(\mu_{0}\right) e^{-x_{0} / \nu_{0}}}{\Lambda^{\prime}\left(\nu_{0}\right) d\left(\nu_{0}^{2}\right)}\right. \\
\left.+\int_{0}^{1}\left(\frac{c \nu^{\prime}}{2}\right)^{2} \frac{\tilde{\varphi}_{+}\left(-\nu^{\prime}\right)}{\Lambda(\infty)\left(\nu_{0}-\nu^{\prime}\right) \gamma\left(\nu^{\prime}\right)} \frac{\varphi_{-\nu}\left(\mu_{0}\right) e^{-x_{0} / \nu^{\prime}}}{\nu^{+}\left(\nu^{\prime}\right) \Lambda^{-}\left(\nu^{\prime}\right)} d \nu^{\prime}\right],
\end{array}\right. \\
& -\frac{c \nu_{0}}{2} \frac{1}{\Lambda(\infty) \gamma_{0} d\left(\nu_{0} \bar{\nu}\right)}, \\
& A(\nu)=S /\left[\left(\nu_{0}-\nu\right) \gamma(\nu) \Lambda^{+}(\nu) \Lambda^{-}(\nu)\right], \\
& S=\left\{\begin{array}{l}
\left(\nu_{0}-\mu_{0}\right) \gamma\left(\mu_{0}\right) \tilde{\varphi}_{\nu}\left(\mu_{0}\right), \\
-c \nu_{0} \nu X\left(-\nu_{0}\right) \tilde{\varphi}_{+}(-\nu), \\
-\left[\nu X\left(-\nu_{0}\right) \tilde{\varphi}_{+}(-\nu) \frac{2 \varphi_{-}\left(\mu_{0}\right) e^{-x_{0} / \nu_{0}}}{\nu_{0} \Lambda^{\prime}\left(\nu_{0}\right) d\left(\nu_{0}^{2}\right)}\right. \\
\left.\quad+\int_{0}^{1}\left(\frac{c \nu^{\prime}}{2}\right)^{2} \frac{\tilde{\varphi}_{\nu}\left(-\nu^{\prime}\right)}{\Lambda(\infty)\left(\nu_{0}-\nu^{\prime}\right) \gamma\left(\nu^{\prime}\right)} \frac{\varphi_{-\nu} \cdot\left(\mu_{0}\right) e^{-z_{0} / \nu^{\prime}}}{\nu^{\prime} \Lambda^{+}\left(\nu^{\prime}\right) \Lambda^{-}\left(\nu^{\prime}\right)} d \nu^{\prime}\right],
\end{array}\right. \\
& -\frac{c \nu}{2} \frac{1}{\Lambda(\infty) \gamma_{0} d\left(\nu_{0} \bar{\nu}\right)} \text {. }
\end{aligned}
$$

The above expansion coefficients for the Milne problem were obtained in a different way by Shure and Natelson.?

The solutions of the four problems are now complete since the angular density is known from (44), (45), and (49) through (52). The neutron densities and net currents, defined by

$$
\begin{aligned}
& \rho(x)=\int_{-1}^{1} \psi(x, \mu) d \mu, \\
& j(x)=\int_{-1}^{1} \mu \psi(x, \mu) d \mu,
\end{aligned}
$$

are easily obtained by integration of (44) and use of (3):

$$
\begin{aligned}
& \rho(x)=\int_{-1}^{1} f(x, \mu) d \mu \\
& +a_{+} e^{-x / r_{0}}+\int_{0}^{1} A(v) e^{-x / v} d v,
\end{aligned}
$$

$$
\begin{aligned}
& j(x)=\int_{-1}^{1} \mu f(x, \mu) d \mu \\
& +(1-c)\left[a_{+} \nu_{0} e^{-x / \nu_{s}}+\int_{0}^{1} A(\nu) \nu e^{-x / v} d \nu\right] .
\end{aligned}
$$

With the above results, one is able to obtain an expression for the Milne problem extrapolation distance, $z_{0}$, that is the distance from the surface of the half-space at which the asymptotic density vanishes. We see that

$$
\rho_{a e}(x) \equiv e^{x / y_{0}}+a_{+} e^{-x / y_{0}}
$$

vanishes at $x=-z_{0}$, with

$$
z_{0}=\frac{1}{2} \nu_{0} \ln \left(-a_{+}^{-1}\right) \text {. }
$$

Equations (49) and (50)(b) and the identity

$$
\tilde{\varphi}_{+}\left(-\nu_{0}\right)=\frac{1}{4} c d\left(\nu_{0}^{2}\right) d\left(-\nu_{0} \bar{\nu}\right) / d\left(\nu_{0} \bar{\nu}\right),
$$

lead to

$z_{0}=\frac{1}{2} y_{0} \ln \left[-X\left(\nu_{0}\right) d\left(\nu_{0} \bar{\nu}\right) / X\left(-\nu_{0}\right) d\left(-\nu_{0} \bar{\nu}\right)\right]$. 


\section{SURFACE QUANTITIES}

For the emerging angular distribution from the half-space, we need to evaluate the expansion (44) for $\mu<0$. Its value for $\mu>0$ is given by Eq. (43). The switching from positive to negative $\mu$ is performed by a trick; we multiply both sides of Eq. (47) by $\tilde{\varphi}_{-\mu^{\prime}}(\mu)\left(\nu_{0}-\mu\right) \gamma(\mu) d \mu$ and integrate. Equation (34) immediately helps us to the general result (for $\mu>0$ ):

$$
\psi(0,-\mu)=f(0,-\mu)+\left(\frac{2}{c \mu}\right)^{2} \Lambda(\infty)\left(\nu_{0}-\mu\right) \gamma(\mu) \int_{0}^{1} \psi\left(\mu^{\prime}\right) \tilde{\varphi}_{-\mu}\left(\mu^{\prime}\right)\left(\nu_{0}-\mu^{\prime}\right) \gamma\left(\mu^{\prime}\right) d \mu^{\prime}
$$

For our four problems, the integral here, as well as the integrals yielding the surface densities and net currents, are all contained in the formulas of Sec. II. We thus arrive at the following results ${ }^{9}$ :

$$
\psi(0,-\mu)= \begin{cases}\frac{2}{c \mu_{0}} \frac{2}{c \mu} \Lambda(\infty)\left(\nu_{0}-\mu_{0}\right) \gamma\left(\mu_{0}\right)\left(\nu_{0}-\mu\right) \gamma(\mu) \tilde{\varphi}_{-\mu_{0}}(\mu), \\ \frac{4 \nu_{0}}{c \mu} \Lambda(\infty) X\left(-\nu_{0}\right)\left(\nu_{0}-\mu\right) \gamma(\mu) \tilde{\varphi}_{+}(\mu), \\ \left(\nu_{0}-\mu\right) \gamma(\mu)\left\{\frac{2}{c \nu_{0}} \frac{2}{c \mu} \frac{2 \Lambda(\infty) X\left(-\nu_{0}\right)}{\Lambda^{\prime}\left(\nu_{0}\right) d\left(\nu_{0}^{2}\right)} \varphi_{+}\left(-\mu_{0}\right) \tilde{\varphi}_{+}(\mu) e^{-x_{0} / \nu_{0}}\right. \\ \left.\quad+\int_{0}^{1} \frac{\varphi_{\nu}\left(-\mu_{0}\right) \tilde{\varphi}_{\nu}(\mu) e^{-x_{0} / \nu}}{\mu\left(\nu_{0}-\nu\right) \gamma(\nu) \Lambda^{+}(\nu) \Lambda^{-}(\nu)} d \nu\right\}, \\ \frac{2}{c \mu} \frac{\left(\nu_{0}-\mu\right) \gamma(\mu)}{\gamma_{0} d\left(\nu_{0} \bar{\nu}\right)} .\end{cases}
$$

Equation (60)(b) was obtained earlier by Shure and Natelson." Equation (60)(a) agrees with the result of Chandrasekhar ${ }^{10}$ after the notation is converted. $^{7}$

The result (60)(a) for the albedo problem shows also that $\psi(0,-\mu) / \mu_{0}$ is a symmetric function of $\mu$ and $\mu_{0}$, in agreement with the reciprocity theorem. ${ }^{10}$

${ }^{10} \mathrm{~S}$. Chandrasekhar, Radiative Transfer (Dover Publications, Inc., New York, 1960).
Moreover, a more general form of this theorem ${ }^{11}$ leads to the conclusion that $\psi(0,-\mu)$ for the Green's function problem differs from the value of $\psi\left(x_{0},-\mu\right) / \mu_{0}$ for the albedo problem only in the interchangement of the variables $\mu$ and $\mu_{0}$. This is verified by Eq. (60)(c) and the albedo problem results.

${ }_{11}$ K. M. Case, Rev. Mod. Phys. 29, 651 (1957). 


\section{THE NONABSORBING MEDIUM}

This case is treated separately because for $c=1$ the two zeros $z= \pm \nu_{0}$ of $\Lambda(z)$ merge at infinity. When $c \rightarrow 1$, we observe that

$$
\begin{aligned}
\nu_{0} & \rightarrow[(1-c)(3-b)]^{-1 / 2}, \\
\gamma_{0} & \rightarrow 1, \\
\gamma_{-1} & \rightarrow \sqrt{3} .
\end{aligned}
$$

The orthogonality relations of Sec. II must be divided by $\nu_{0}$ before taking the limit $c \rightarrow 1$. With the addition of some auxiliary formulas, the set of needed relations becomes

$\int_{0}^{1} \varphi_{v}(\mu) \varphi_{p} \cdot(\mu) \gamma(\mu) d \mu$

$$
=\gamma(\nu) \Lambda^{+}(\nu) \Lambda^{-}(\nu) \delta\left(\nu-\nu^{\prime}\right),
$$

$\int_{0}^{1} \varphi_{-,}(\mu) \varphi_{\nu},(\mu) \gamma(\mu) d \mu=\frac{3}{4} \nu^{2} \varphi_{\nu},(-\nu) / \gamma(\nu)$,

$\int_{0}^{1} \varphi_{-\nu}(\mu) \varphi_{-,},(\mu) \gamma(\mu) d \mu$

$$
=\frac{3}{4} \nu^{\prime} \varphi_{\nu}\left(\nu^{\prime}\right)\left[\frac{\nu^{\prime}}{\gamma\left(\nu^{\prime}\right)}-\frac{\nu}{\gamma(\nu)}\right],
$$

$\int_{0}^{1} \varphi_{\nu}(\mu) \gamma(\mu) \mu d \mu=-\frac{1}{2} \nu$

$\int_{0}^{1} \varphi_{->}(\mu) \gamma(\mu) \mu d \mu=\frac{1}{2} \nu-\frac{3}{4} \nu^{3} / \gamma(\nu)$,

$\int_{0}^{1} \varphi_{\nu}(\mu) \gamma(\mu) d \mu=0$

$\int_{0}^{1} \varphi_{-,}(\mu) \gamma(\mu) d \mu=\frac{3}{4} \nu^{2} / \gamma(\nu)$

$\int_{0}^{1} \varphi_{\nu}(\mu) \gamma(\mu) \frac{d \mu}{\mu}=\frac{1}{2} \sqrt{3}$,

$\int_{0}^{1} \varphi_{-\nu}(\mu) \gamma(\mu) \frac{d \mu}{\mu}=\frac{1}{2} \sqrt{3}-\frac{3}{4} \nu / \gamma(\nu)$.

Nothing here depends upon the anisotropy parameter $b$. All the functions involved and all the formulas are the same as for isotropic scattering.

Since the two discrete modes used before have now become identical, we choose a new basis which includes the following two solutions of the homogeneous transport equation:

$\psi_{1}(x, \mu)=\frac{1}{2}, \quad \psi_{2}(x, \mu)=\frac{3}{2}\left[\left(1-\frac{1}{3} b\right) x-\mu\right]$.

The expansion (44) is replaced by

$$
\begin{aligned}
& \psi(x, \mu)=f(x, \mu) \\
& \quad+\frac{1}{2} a_{1}+\int_{0}^{1} A(\nu) \varphi_{\nu}(\mu) e^{-x / \nu} d \nu .
\end{aligned}
$$

For the (a) albedo, (b) Milne, and (c) Green's function problems, $f(x, \mu)$ will be taken as follows:

$$
f(x, \mu)=\left\{\begin{array}{l}
0, \\
\psi_{2}(x, \mu), \\
G_{\infty}^{\prime}\left(x_{0}, \mu_{0} \rightarrow x, \mu\right) .
\end{array}\right.
$$

By the choice of $(75)(b)$, the solution of the Milne problem has been renormalized to unit net current. The Green's function for the infinite medium is chosen such that its value is finite at $x \rightarrow+\infty$, namely

$G_{\infty}^{\prime}\left(x_{0}, \mu_{0} \rightarrow x, \mu\right)= \begin{cases}\psi_{2}\left(x_{0},-\mu_{0}\right)+\cdots, & x>x_{0}, \\ \psi_{2}(x, \mu)+\cdots, & x<x_{0},\end{cases}$

where the dots indicate the same integral as in Eq. (46).

The final results for the three problems then follow through application of the above formulas and are given in the following list:

$$
\begin{aligned}
& a_{1}=\left\{\begin{array}{l}
2 \gamma\left(\mu_{0}\right), \\
3 \bar{\nu}, \\
3 \bar{\nu}-\frac{3}{2} \int_{0}^{1} \frac{\nu^{\prime} \varphi_{-\nu} \cdot\left(\mu_{0}\right) e^{-x_{0} / \nu^{\prime}}}{\gamma\left(\nu^{\prime}\right) \Lambda^{+}\left(\nu^{\prime}\right) \Lambda^{-}\left(\nu^{\prime}\right)} d \nu^{\prime},
\end{array}\right. \\
& A(\nu)=\left\{\begin{array}{l}
\gamma\left(\mu_{0}\right) \varphi_{v}\left(\mu_{0}\right) / \gamma(\nu) \Lambda^{+}(\nu) \Lambda^{-}(\nu), \\
-3 v / 4 \gamma(\nu) \Lambda^{+}(\nu) \Lambda^{-}(\nu) \\
-\frac{3}{4 \gamma(\nu) \Lambda^{+}(\nu) \Lambda^{-}(\nu)}\{\nu \\
\left.+\int_{0}^{1} \frac{\nu^{\prime} \varphi_{v}\left(-\nu^{\prime}\right) \varphi_{-\nu}\left(\mu_{0}\right) e^{-x_{0} / \nu^{\prime}}}{\gamma\left(\nu^{\prime}\right) \Lambda^{+}\left(\nu^{\prime}\right) \Lambda^{-}\left(\nu^{\prime}\right)} d \nu^{\prime}\right\},
\end{array}\right.
\end{aligned}
$$

$$
\begin{gathered}
\rho(0)=\left\{\begin{array}{l}
(2 / \sqrt{3}) \gamma\left(\mu_{0}\right) / \mu_{0} \\
\sqrt{3}, \\
\sqrt{3}\left\{1+\frac{1}{2} \int_{0}^{1} \frac{\varphi_{-\nu}\left(\mu_{0}\right) e^{-x_{0} / \nu}}{\gamma(\nu) \Lambda^{+}(\nu) \Lambda^{-}(\nu)} d \nu\right.
\end{array}\right\} \\
j(0)=\left\{\begin{array}{cc}
0, & \text { (a) } \\
-1 . & \text { (b) },(\mathrm{c})
\end{array}\right.
\end{gathered}
$$

Again the results (most of them known) are the same as for isotropic scattering. The only term dependent upon $b$ is the $f(x, \mu)$ in the cases $(b)$ and $(c)$. 
This agrees with the general observation ${ }^{7}$ that $\psi(x, \mu)$ and the corresponding solution for $b=0$ differ only by a term $\frac{1}{2} b j x$ if the medium is nonabsorbing.

The asymptotic part of the density for the Milne problem is

$$
\rho_{a s}(x) \equiv(3-b) x+3 \bar{\nu}
$$

so

$$
z_{0}=\left(1-\frac{1}{3} b\right)^{-1} \bar{\nu} .
$$

This result is well-known: $\left(1-\frac{1}{3} b\right)^{-1}$ is the transport mean free path and $\bar{\nu}=0.710446$.

\section{ACKNOWLEDGMENTS}

We are indebted to Professor G. C. Summerfield and Professor F. C. Shure for helpful discussions. 